\title{
6 Klassifizierung mathematischer Handlungsaspekte im optes-Vorkurs
}

\author{
Anna-Katharina Roos, Hans-Georg Weigand, Jan Wörler
}

Welches mathematische Wissen und Können, welche Fähigkeiten und Fertigkeiten benötigen Studierende eines technischen Studiengangs? Welche Definitionen mathematischer Begriffe sollten sie kennen, welche Rechenverfahren richtig anwenden können, welche mathematischen Beweise sollten sie wiedergeben, nachvollziehen oder selbst finden können? Diese Fragen standen am Anfang der Erstellung des optes-Onlinekurses Mathematik.

\section{1 optes-Onlinekurs Mathematik}

Für viele Lernende stellt sich der Übergang von der Schule zur Universität als problembehaftet heraus (Gueudet 2008). Um den Schwierigkeiten der Lernenden zu begegnen, wurde inzwischen eine Vielzahl an mathematischen Vor- und Brückenkursen entwickelt (Beispiele findet man in Hoppenbrock et al. 2016; Roth et al. 2015). Deren Ziel ist es, die Lücke zwischen Schule und Hochschule zu reduzieren. Üblicherweise finden die Kurse vor dem Start des ersten Semesters statt. Neben Lernenden, die sich für ein Mathematikstudium entschieden haben, ist Mathematik auch für Studierende eines technischen Studiengangs eine wesentliche Grundvoraussetzung für ein erfolgreiches Studium. Für diese wird Mathematik vor allem als Werkzeug für andere Fächer wie Physik, Chemie, Elektrotechnik etc. genutzt (Kortemeyer 2018, S. 11). Hier ist eine passende Problem- und Lösungsformulierung nur mit Hilfe der mathematischen Sprache möglich und mathematische Kompetenzen sind unabdingbar, um Aufgaben in Fächern wie Elektrotechnik zu lösen (Kortemeyer 2018, S. 5).

Genau für diese Zielgruppe ist der optes-Onlinekurs Mathematik entwickelt worden. Er beabsichtigt eine Wiederholung und Festigung der schulischen Inhalte, aufbereitet für den Übergang von der Schule zur Hochschule. Im Fokus stehen dabei zukünftige Studierende eines technischen Studiengangs an Hochschulen und dualen Hochschulen in ganz Deutschland. Da diese heutzutage eine Vielzahl verschiedener (Schul-)Ausbildungen zur Erlangung der Hochschulzugangsberechtigung mit sich bringen, stellen sie hinsichtlich des mathematischen Vorwissens beim Eintritt an die Universität eine sehr heterogene Gruppe dar (z. B. Blömeke 2016; de Guzman et al. 1998). Die Heterogenität stellt insbesondere die Dozent*innen der Anfangsvorlesungen in Mathematik vor große Herausforderungen. Diesen verschiedenartigen mathematischen Wissensständen entgegenzuwirken und den

Die Originalversion dieses Kapitels wurde revidiert. Ein Erratum ist verfügbar unter https://doi.org/10.1007/978-3-658-31279-4_32 
Studierenden bereits vor Studienbeginn die Möglichkeit zu geben, solide Grundlagen für die mathematischen Themen ihrer zukünftigen Ausbildung vorzubereiten, ist ein wesentliches Ziel des optes-Onlinekurses. Der Kurs soll Inhalte, die relevant für ein Hochschulstudium sind oder als relevant angesehen werden, aufgreifen, wiederholen und vertiefen. Eine Quelle für zentrale und wichtige Inhalte der Schulmathematik findet sich im sogenannten cosh-Katalog (2014), den Dozent*innen von Hochschulen sowie Lehrkräfte an Gymnasien vor allem aus dem Raum Baden-Württemberg zusammengestellt haben.

Die mathematischen Inhalte des optes-Onlinekurses wurden in sechs sogenannte Lernzielorientierte Kurse (LoKs) gegliedert:

Tabelle 1: Inhalte des optes-Onlinekurses gegliedert in sechs Lernzielorientierte Kurse

\begin{tabular}{ll}
\hline LoK 1 & Arithmetik \\
LoK 2 & Gleichungen und Ungleichungen \\
LoK 3 & Potenzen, Wurzeln, Logarithmen \\
LoK 4 & Funktionen \\
LoK 5 & Geometrie \\
\hline LoK 6 & Trigonometrie \\
\hline
\end{tabular}

Diese LoKs bestehen sowohl aus erklärenden Texten und Übungsaufgaben, ähnlich einem traditionellen Mathematikbuch, die aber mit interaktiven Elementen wie bspw. Geogebra-Applets (siehe Kapitel 14) ergänzt wurden. Darüber hinaus können Studierende in verschiedenen Tests und Trainings (siehe Kapitel 3) mit auf die jeweiligen Inhalte abgestimmten Aufgaben ihr Wissen überprüfen und üben. Basierend auf den Bearbeitungen dieser Aufgaben soll den Lernenden im Anschluss ein sinnvolles und hilfreiches Feedback über ihr dort gezeigtes mathematisches Können im Kontext von sogenannten E-Portfolios (siehe Kapitel 5) gegeben werden. Die Existenz eines Modells, um Studierenden hilfreiche und detaillierte Feedbacks geben zu können, ist somit ein wichtiger Bestandteil auf der Meta- oder Hintergrundebene eines LoKs.

\subsection{Einführung eines Kompetenzmodells in optes}

Um dem Charakter der Mathematik und den Anforderungen innerhalb eines ingenieur- bzw. naturwissenschaftlichen Studiums gerecht zu werden, darf ein Feedback zum mathematischen Wissen und Können nicht nur auf der inhaltlichen Ebene, sondern sollte auch auf einer prozessbezogenen Ebene erfolgen. Leztere bezieht sich auf mathematische Handlungen wie etwa Beweisen, Darstellen oder Modellieren. Es gilt also nicht nur die Frage zu stellen, welche Inhalte vermittelt werden sollen, sondern es muss zusätzlich berücksichtigt werden, welche typisch 
mathematischen Arbeits- und Handlungsweisen Lernende entwickeln sollen. Üblicherweise werden diese Ziele heute mit dem Kompetenzbegriff umschrieben. Weinert (2001) charakterisiert Kompetenzen als ,die bei Individuen verfügbaren oder durch sie erlernbaren kognitiven Fähigkeiten und Fertigkeiten, um bestimmte Probleme zu lösen, sowie die damit verbundenen motivationalen, volitionalen und sozialen Bereitschaften und Fähigkeiten, um Problemlösungen in variablen Situationen erfolgreich und verantwortungsvoll nutzen zu können“ (Weinert 2001, S. 27 f.).

In dem optes-Onlinekurs haben wir ein Kompetenzmodell eingeführt, das vorrangig auf das Schweizer HarmoS-Modell (Schweizerische Konferenz der kantonalen Erziehungsdirektoren 2011) zurückgreift. Dieses wurde als Basis gewählt, da es zum einen eine Fortentwicklung der deutschen KMK-Standards und mittlerweile theoretisch und empirisch gut verankert ist (z. B. Linneweber-Lammerskitten 2014). Zum anderen haben wir es gewählt, weil es für unsere Zwecke passend die mathematischen Fähigkeiten und Fertigkeiten neben den inhaltlichen Bereichen in Form von Handlungsaspekten repräsentiert, deren Ausbildung wir uns von zukünftigen Ingenieur*innen und Naturwissenschaftler*innen wünschen.

\subsubsection{Das schweizerische HarmoS-Modell}

Das schweizerische HarmoS-Modell hat sich ,von Kompetenzmodellen aus anderen Ländern bzw. internationalen Projekten (NCTM, PISA, KMK u. a .m.), aber auch von den Besonderheiten der Schweiz (Lehrplanvergleich, Kultur- und Sprachunterschiede) leiten lassen" (Schweizerische Konferenz der kantonalen Erziehungsdirektoren 2011, S. 5). Dieses Kompetenzmodell unterteilt sich in Handlungsaspekte (prozessbezogene Kompetenzen), inhaltsbezogene Kompetenzen, verschiedene Kompetenzniveaus, eine Entwicklungsdimension und eine nichtkognitive Dimension (insbesondere motivationale und soziale Facetten) (Schweizerische Konferenz der kantonalen Erziehungsdirektoren 2011, S. 6). Für uns interessant und relevant sind dabei vor allem die Ausarbeitung der einzelnen Handlungsaspekte in der Jahrgangsstufe 11.

Das allgemeine Zusammenwirken der Handlungsaspekte (prozessbezogene Kompetenzen) und der Kompetenzbereiche (inhaltsbezogene Kompetenzen) verdeutlicht Abbildung 1. 


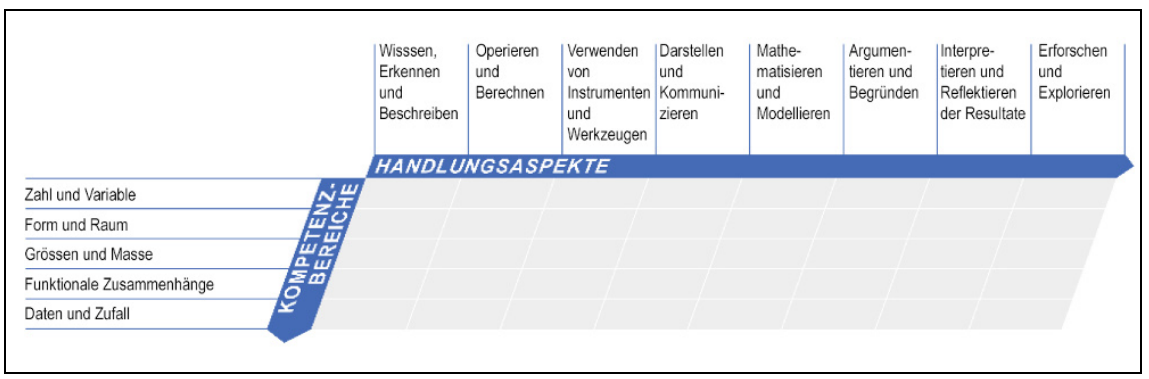

Abbildung 1: Kompetenzbereiche und Handlungsaspekte im HarmoS-Modell (Schweizerische Konferenz der kantonalen Erziehungsdirektoren 2011, S. 7)

\subsubsection{Das Kompetenzmodell in optes}

Aufbauend auf den Überlegungen des HarmoS-Modells wurde für den optes-Kurs ein eigenes Kompetenzmodell basierend auf prozessbezogenen und inhaltsbezogenen Kompetenzen entwickelt. Den fünf inhaltsbezogenen Kompetenzen des HarmoS-Modells entsprechend wurden sechs Basis-LoKs entwickelt und zu fünf Handlungsaspekten in Beziehung gestellt.

Das hier verwendete Kompetenzmodell sieht vor dem Hintergrund theoretischer Überlegungen aktuell folgendermaßen aus:

Tabelle 2: Inhaltsbereiche und Handlungsaspekte in optes

\begin{tabular}{|c|c|c|c|c|c|c|}
\hline \multirow{8}{*}{ 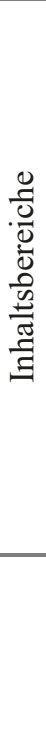 } & $\begin{array}{l}\text { LoK 1: } \\
\text { Arithmetik }\end{array}$ & & & & & \\
\hline & $\begin{array}{l}\text { LoK 2: } \\
\text { Gleichungen/ } \\
\text { Ungleichun- } \\
\text { gen }\end{array}$ & & & & & \\
\hline & $\begin{array}{l}\text { LoK 3: } \\
\text { Potenzen, } \\
\text { Wurzeln, } \\
\text { Logarithmen }\end{array}$ & & & & & \\
\hline & $\begin{array}{l}\text { LoK 4: } \\
\text { Funktionen }\end{array}$ & & & & & \\
\hline & $\begin{array}{l}\text { LoK 5: } \\
\text { Geometrie }\end{array}$ & & & & & \\
\hline & $\begin{array}{l}\text { LoK 6: } \\
\text { Trigonometrie }\end{array}$ & & & & & \\
\hline & & $\begin{array}{l}\text { Wiederge- } \\
\text { ben, Erken- } \\
\text { nen, Be- } \\
\text { schreiben }\end{array}$ & $\begin{array}{l}\text { Operie- } \\
\text { ren und } \\
\text { Berech- } \\
\text { nen }\end{array}$ & $\begin{array}{c}\text { Darstel- } \\
\text { lungen } \\
\text { verwenden }\end{array}$ & $\begin{array}{l}\text { Mathema- } \\
\text { tisieren }\end{array}$ & $\begin{array}{l}\text { Argumen- } \\
\text { tieren und } \\
\text { Begründen }\end{array}$ \\
\hline & & \multicolumn{5}{|c|}{ Handlungsaspekte } \\
\hline
\end{tabular}


Die Einteilung der Handlungsaspekte zu den jeweiligen Inhaltsbereichen orientiert sich dabei stark an den Einteilungen des HarmoS-Modells für die 11. Jahrgangsstufe (Schweizerische Konferenz der kantonalen Erziehungsdirektoren 2011, S. 18 ff.), wobei wir folgende Zuweisungen vorgenommen haben:

Tabelle 3: Zuweisung der Beschreibungen der Handlungsaspekte im HarmoS-Modell zu den LoKs in optes

\begin{tabular}{|l|l|}
\hline HarmoS-Modell & optes-LoKs \\
\hline Zahl und Variable & 1 Arithmetik \\
Zahl und Variable & 2 Gleichungen und Ungleichungen \\
Zahl und Variable & 3 Potenzen, Wurzeln, Logarithmen \\
Funktionale Zusammenhänge & 4 Funktionen \\
$\begin{array}{l}\text { Form und Raum } \\
\text { Selbst entwickelt, teilweise funktionale } \\
\text { Zusammenhänge }\end{array}$ & 5 Geometrie \\
\hline
\end{tabular}

Die Handlungsaspekte „Verwenden von Instrumenten und Werkzeugen“, „Interpretieren und Reflektieren der Resultate“, „Erforschen und Explorieren“ wurden in unserem Modell nicht berücksichtigt, da deren Umsetzungen unter den Gegebenheiten eines Online-Brückenkurses nur schwer - zudem kaum in dem begrenzten Zeitumfang - zu realisieren sind. Aus dem gleichen Grund wurde der Handlungsaspekt „Darstellen und Kommunizieren“ auf „Darstellungen verwenden“ und der Handlungsaspekt „Mathematisieren und Modellieren“ auf „Mathematisieren“ eingeschränkt. Tabelle 4 gibt einen Überblick über die vorgenommenen Abänderungen.

Tabelle 4: Anpassung der Handlungsaspekte des HarmoS-Modells in den optes-Kursen

\begin{tabular}{|l|l|}
\hline HarmoS-Modell & optes-LoKs \\
\hline Wissen, Erkennen und Beschreiben & Wiedergeben, Erkennen, Beschreiben \\
Operieren und Berechnen & Operieren und Berechnen \\
Darstellen und Kommunizieren & Darstellungen verwenden \\
Mathematisieren und Modellieren & Mathematisieren \\
Argumentieren und Begründen & Argumentieren und Begründen \\
Wissen, Erkennen und Beschreiben & Wiedergeben, Erkennen, Beschreiben \\
\hline
\end{tabular}




\subsubsection{Aufgaben im Rahmen des Kompetenzmodells}

Wir gehen von der folgenden These aus: „Die Brücke zwischen theoretischen Kompetenzmodellen und ihrer empirischen Erfassung bildet die Konkretisierung der Theorieelemente durch Aufgabensituationen“ (Leuders 2014, S. 13). Dementsprechend wurde basierend auf unserem Kompetenzmodell ein Kodierleitfaden für jeden LoK entwickelt. Alle Aufgaben der Trainings und der Tests wurden mit diesem höchstens zwei Handlungsaspekten unseres Kompetenzmodells zugeordnet.

Nachdem Lernende einen Test oder ein Training beendet haben, wird ihnen für jeden einzelnen Handlungsaspekt der Anteil an richtig gelösten Aufgaben im EPortfolio angezeigt. Die folgende Grafik zeigt dies beispielhaft:

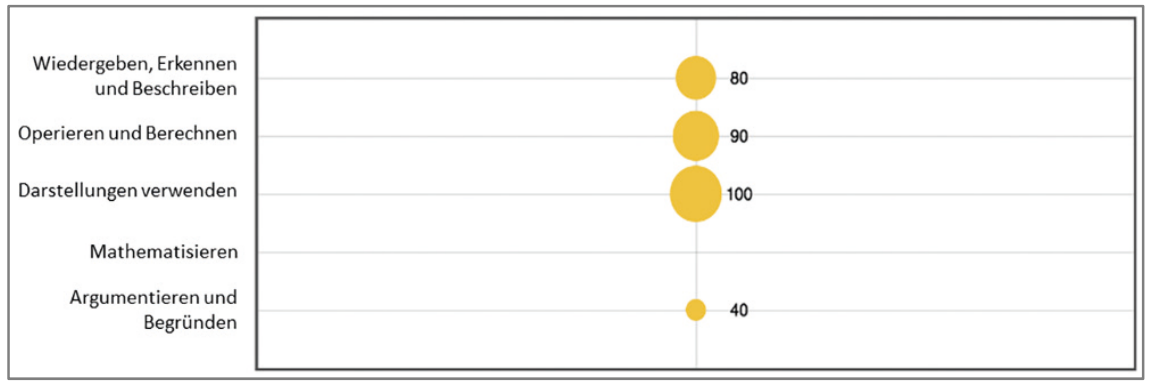

Abbildung 2: Darstellung der Ausprägung der Handlungsaspekte im E-Portfolio

Ausgehend von diesen Rückmeldungen an die Lernenden haben diese in einem sogenannten „Rückweg“ die Möglichkeit, speziell Aufgaben zu einem Handlungsaspekt, mit dessen Ausprägung sie noch nicht zufrieden sind, zu üben. Dazu können die Lernenden sich in einem LoK beispielsweise gezielt Aufgaben zum Mathematisieren, Argumentieren oder zu jedem anderen Handlungsaspekt geben lassen. Dies bietet die Gelegenheit, individuell an möglichen Schwächen zu arbeiten.

\subsection{Grundzüge und Vorbereitungen einer empirischen Validierung des Kompetenzmodells}

Im Anschluss an die Einführung und die Pilotierung dieses Modells an unseren Partnerhochschulen hat sich die Frage gestellt, inwieweit die Rückmeldung an Lernende im Rahmen des Kompetenzmodells den tatsächlichen Wissens- bzw. Kompetenzstand der Studierenden erfasst. Insbesondere sind wir der Frage nachgegangen: Wie valide werden die jeweiligen Kompetenzen gemessen? Eine umfassende quantitative Validitätsüberprüfung des Kompetenzmodells war allerdings aufgrund zeitlicher Restriktionen noch nicht möglich. Wir entschieden uns deshalb 
dafür, in einer explorativen qualitativen Studie zunächst Hinweise auf die Passung oder Validität unseres Modells zu erhalten. Darüber hinaus interessierten wir uns im Hinblick auf die mathematischen Veranstaltungen in der Eingangsphase auch für Gründe für eventuelle existierende Mängel bei Studienanfänger*innen. Dabei standen die folgenden Fragen im Mittelpunkt:

FF1: Lassen sich die Rückmeldungen unseres Kompetenzmodells hinsichtlich der einzelnen Handlungsaspekte empirisch bestätigen?

FF2: Worin liegen die Schwierigkeiten von Studienanfänger*innen bei einzelnen Handlungsaspekten?

FF3: Lassen sich Gründe für vorhandene Schwierigkeiten erkennen?

\subsubsection{Methodik der Untersuchung}

Um unser Kompetenzmodell zu überprüfen, haben wir uns exemplarisch zunächst auf den Inhaltsbereich Arithmetik konzentriert. Arithmetik ist der erste LoK und damit für viele der Einstieg in den optes-Onlinekurs.

Um die Forschungsfragen zu beantworten, haben wir uns für Interviews entschieden. Bortz und Döring beschreiben:

Am Beginn der theoretischen Auseinandersetzung mit einem wenig erforschten Thema stellt sich oft die Frage, welche Aspekte, Facetten oder Komponenten überhaupt von Bedeutung sind; gesucht wird also zunächst eine Auflistung der wichtigsten Elemente des untersuchten Phänomens, d. h. ein Inventar. Hierfür eigenen sich teilstrukturierte Interviews mit offenen Fragen, deren Ergebnisse inhaltsanalytisch auszuwerten sind. (Bortz und Döring 2006, S. 381)

Im Sommersemester 2019 wurde an einer unserer Partnerhochschulen, der Dualen Hochschule Baden-Württemberg (DHBW Mosbach, ein Pilot für ein Online-Training mit Aufgaben aus der Arithmetik durchgeführt. Unter den aktiven Studierenden hat sich eine Gruppe von sechs Studierenden bereit erklärt, an Interviews teilzunehmen. Alle befragten Studierenden hatten dabei für die jeweils betrachteten Handlungsaspekte mindestens drei Fragen bearbeitet und alle Handlungsaspekte wurden durch mehrere Studierende abgedeckt.

Während der Interviews wurden den Studierenden von ihnen (teilweise falsch bearbeitete Aufgaben nochmal vorgelegt und nachgefragt, an welcher Stelle Schwierigkeiten aufgetreten waren. Außerdem wurden ihnen zusätzliche Fragen gestellt, um weitergehend zu sehen, wie sie, im Rahmen des jeweils angesprochenen Handlungsaspekts, neue Aufgaben bearbeiten. Es wurde also für alle Studierenden ein jeweils individueller Leitfaden im Vorfeld angelegt. Trotzdem wurde darauf geachtet, genug Raum für spontane Nachfragen der Interviewenden zu lassen. Ein Interview dauerte circa 20 Minuten. 


\subsubsection{Ergebnisse}

Die von uns gefundenen Ergebnisse werden hier exemplarisch anhand einiger Interviewausschnitte aufgezeigt und den einzelnen Handlungsaspekten zugeordnet. Zunächst ein allgemeiner Überblick der Ergebnisse der sechs hier vorgestellten Studierenden ${ }^{1}$.

Tabelle 5: Rückmeldung des Kompetenzmodells in optes an die Teilnehmer*innen des Interviews

\begin{tabular}{|c|c|c|c|}
\hline $\begin{array}{l}\text { ELLA } \\
\text { Argumentieren: } \\
\text { Operieren: } \\
\text { Wiedergeben: } \\
\text { Mathematisieren: } \\
\text { Darstellungen ver- } \\
\text { wenden: }\end{array}$ & $\begin{array}{l}0 \text { von } 1(0 \%) \\
7 \text { von } 9(78 \%) \\
5 \text { von } 6(83 \%) \\
2 \text { von } 5(40 \%) \\
5 \text { von } 5(100 \%)\end{array}$ & $\begin{array}{l}\text { ADAM } \\
\text { Argumentieren: } \\
\text { Operieren: } \\
\text { Wiedergeben: } \\
\text { Mathematisieren: } \\
\text { Darstellungen ver- } \\
\text { wenden: }\end{array}$ & $\begin{array}{l}0 \text { von } 4(0 \%) \\
11 \text { von } 21(52 \%) \\
3 \text { von } 3(100 \%) \\
8 \text { von } 16(50 \%) \\
3 \text { von } 3(100 \%)\end{array}$ \\
\hline Gesamt: & 19 von $24(79 \%)$ & Gesamt: & 23 von $40(58 \%)$ \\
\hline $\begin{array}{l}\text { RON } \\
\text { Argumentieren: } \\
\text { Operieren: } \\
\text { Wiedergeben: } \\
\text { Mathematisieren: } \\
\text { Darstellungen ver- } \\
\text { wenden: }\end{array}$ & $\begin{array}{l}3 \text { von } 4(75 \%) \\
16 \text { von } 32(50 \%) \\
4 \text { von } 6(67 \%) \\
6 \text { von } 17(35 \%)\end{array}$ & $\begin{array}{l}\text { BASIL } \\
\text { Argumentieren: } \\
\text { Operieren: } \\
\text { Wiedergeben: } \\
\text { Mathematisieren: } \\
\text { Darstellungen ver- } \\
\text { wenden: }\end{array}$ & $\begin{array}{l}1 \text { von } 4(25 \%) \\
19 \text { von } 29(66 \%) \\
6 \text { von } 8(75 \%) \\
5 \text { von } 9(56 \%) \\
3 \text { von } 3(100 \%)\end{array}$ \\
\hline Gesamt: & 28 von $54(52 \%)$ & Gesamt: & 30 von $46(65 \%)$ \\
\hline $\begin{array}{l}\text { MALIN } \\
\text { Argumentieren: } \\
\text { Operieren: } \\
\text { Wiedergeben: } \\
\text { Mathematisieren: } \\
\text { Darstellungen ver- } \\
\text { wenden: }\end{array}$ & $\begin{array}{l}2 \text { von } 4(50 \%) \\
14 \text { von } 25(56 \%) \\
6 \text { von } 11(55 \%) \\
3 \text { von } 7(43 \%)\end{array}$ & $\begin{array}{l}\text { MIRO } \\
\text { Argumentieren: } \\
\text { Operieren: } \\
\text { Wiedergeben: } \\
\text { Mathematisieren: } \\
\text { Darstellungen ver- } \\
\text { wenden: }\end{array}$ & $\begin{array}{l}0 \text { von } 1(0 \%) \\
13 \text { von } 18(72 \%) \\
4 \text { von } 4(100 \%) \\
7 \text { von } 15(47 \%)\end{array}$ \\
\hline Gesamt: & 24 von $46(52 \%)$ & Gesamt: & 22 von $33(67 \%)$ \\
\hline
\end{tabular}

1 Hier anonymisiert mit geänderten Namen vorgestellt. 


\section{Mathematisieren}

Eine der Aufgaben, die Ron im Vorfeld der Interviews zum Handlungsaspekt Mathematisieren bearbeitet hatte, ist die folgende:

Eine Pumpe kann einen Pool in 12 Stunden leer pumpen, eine andere Pumpe benötigt dafür 15 Stunden. Beide Pumpen zusammen haben 5 Stunden lang gearbeitet.

Welcher Anteil $x$ des Pools ist danach noch mit Wasser gefüllt?

Lösung: $x=\frac{1}{4}$

Aufgabe 1: Eine Aufgabe zum Handlungsaspekt Mathematisieren

Ron hatte diese Aufgabe falsch bearbeitet und erklärt dazu im Interview:

Ron: „Da fehlt einem auch wieder der Ansatz. Wie fange ich an mit der Aufgabe? Ja. Also ich muss ja irgendwie wieder eine Gleichung aufstellen. Also die Gleichung lösen ist schon wieder kein Problem. Aber die Gleichung aufstellen, aus einer Textaufgabe eine Gleichung rausfinden, stellt sich mir echt schwierig dar."

I: „Also generell jetzt, oder?“

Ron: „Allgemein. Also ja, tatsächlich. Das ist schon eher nicht so gut bei mir.“

I: „Was würdest du sagen ist in Mathe dann eher so gut bei dir? Also was ist gut, was ist weniger gut?"

Ron: „Ja, lösen der Gleichung, das kriege ich dann schon hin. Lösen der Gleichungen.“

Ron trennt hier verschiedene Fähigkeiten beim Lösen mathematischer Aufgaben: Zum einen sagt er, einen Ansatz aufzustellen falle ihm schwer. Dieser Aspekt fällt in unserem Kompetenzmodell unter den Handlungsaspekt Mathematisieren, also das Übersetzen verbal schriftlich gegebener Situationen in eine symbolische Formel. Zum anderen spricht er auch das Lösen mathematischer Gleichungen an. Dieser Prozess des algorithmischen Anwendens mathematischer Rechenverfahren fällt in unserem Kompetenzmodell unter den Handlungsaspekt des Operierens und Berechnens. Dazu reflektiert Ron, dass ihm dies weniger Schwierigkeiten bereite.

Rons Beispiel macht dabei deutlich, dass eine getrennte Betrachtung des Mathematisierens vom Aspekt des reinen Operierens sinnvoll ist.

Auch ein Teil der anderen Teilnehmer*innen, Ella, Miro und Adam, beschreiben ihre Schwierigkeiten ähnlich. Malin bringt seine Gedanken auf den Punkt: „Da ist irgendwie kein Ansatz da.“

Regelmäßig wurden ähnliche Begründungen von den Lernenden für Probleme mit den von uns zum Handlungsaspekt Mathematisieren gegebenen Aufgaben genannt.

Die Einstufung der Studierenden durch unser Kompetenzsystem scheint insbesondere bei diesem Handlungsaspekt sehr gut zu passen. 


\section{Darstellungen verwenden}

Ron hatte die folgende Aufgabe (2) vor dem Interview falsch bearbeitet.

a)

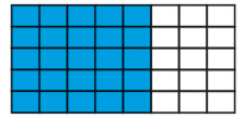

c)

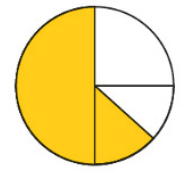

b)

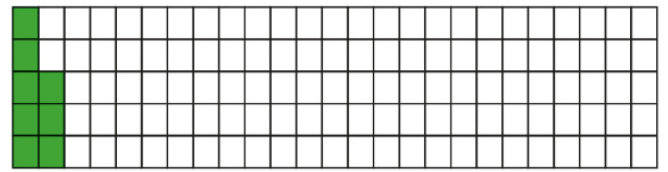

d)

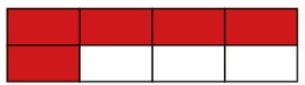

e)

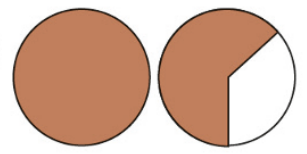

Aufgabe 2: Eine Aufgabe zum Handlungsaspekt Darstellungen verwenden

Im Zuge des Interviews wird ihm diese erneut vorgelegt und er soll seine Gedankengänge erläutern:

Ron: „Wenn ich das hier schon sehe: 25 Achtel, Fünftel. Dann hört es in mir schon wieder auf, weil ich mir das nicht vorstellen kann. Manche Rechenregeln von früher kenn ich einfach nicht mehr. Und deshalb habe ich mit dem Begriff ,25/8 durch 5' nichts anfangen können. Das ist oft bei mir der Fall, dass ich Rechenregeln von früher nicht mehr weiß.“

I: „Das heißt: Dein Problem an der Aufgabe ist eigentlich der Ausdruck.“

Ron: ,Ja.“

I: „Okay. Wenn da jetzt stehen würde,$\frac{5}{8}$ ‘ einfach. Und du sollst die Aufgabe für $\frac{5}{8}$ lösen. Kannst du die lösen?"“

Der Interviewer löst hier den gegebenen Doppelbruch in einen einfachen Bruch auf.

Ron: „Nein, ich glaube nicht, weil ich mir das nicht vorstellen kann, wie das aussieht.“ I: , ,5/8?“

Ron: „Ja. Ich weiß nicht, wie man das in einer Grafik ausdrückt: $\frac{5}{8}$. Also klar, man kennt so die klassischen $\frac{3}{4}, \frac{1}{4}, \frac{2}{4}$. “

I: „Wie sieht denn das aus? $\frac{3}{4}, \frac{1}{4}$ ?“ 
Ron: „Ja $\frac{3}{4}$ ist halt im Kreis (zeichnet) das hier. Aber $\frac{5}{8}$ kann ich mir als Grafik nicht vorstellen."

I: „Okay, hast du von $\frac{5}{8}$ auf dem Zahlenstrahl eine Vorstellung oder auch nicht?““

Ron: „Nein.“

I: „Einfach nur: Du siehst $\frac{5}{8}$ und (abgebrochen)“

Ron: ,,$\frac{5}{8}$ ist bei mir eine Zahl, die kommt nur in den Taschenrechner.“

Rons Erklärung macht bei dieser Aufgabe deutlich, welche Probleme er mit der Darstellung von Bruchzahlen hat. Das Kompetenzmodell hat ihm also richtig - im Gegensatz zu den anderen Teilnehmer*innen - noch Verbesserungen hinsichtlich dieses Handlungsaspekts attestiert. Aber auch in Bezug auf den Aspekt „Wiedergeben, Erkennen, Beschreiben“(Umwandlung eines Doppelbruchs) sieht man bei ihm Lücken.

Die Teilnehmer*innen Miro, Basil, Ella und Adam, die von unserem Kompetenzsystem einen sehr guten Umgang mit mathematischen Darstellungen ausgewiesen bekommen haben, können auch mit der ihnen gegebenen Alternativfrage sehr gut arbeiten.

Insgesamt scheint das Modell hinsichtlich des Aspekts „Darstellungen verwenden" hilfreiche Einstufungen gegeben zu haben. Allerdings bleibt insbesondere hier zu beachten, dass von den Teilnehmer*innen relativ wenige Aufgaben bearbeitet wurden.

\section{Argumentieren und Begründen}

Alle von uns gewählten Interviewteilnehmer*innen hatten von unserem Kompetenzsystem eine mangelhafte Ausprägung hinsichtlich „Argumentieren und Begründen“ zugewiesen bekommen. Während des Interviews wurde ihnen die folgende, neue Frage zum Argumentieren gestellt, um zu sehen, wie sie dabei arbeiten: 


\section{Alternativfrage:}

Erklären Sie die folgenden durchgeführten Rechenschritte und bewerten Sie die Lösung:

Lösen Sie für $x \in \mathbb{R}$ die Gleichung $\frac{1}{2}(10 x+8)=4+2 x$.

$$
\begin{aligned}
& \frac{1}{2}(10 x+8)=4+2 x \\
= & 5 x+4=4+2 x \\
= & 5 x=2 x \\
= & 5=2
\end{aligned}
$$

Es gibt keine Lösung der Gleichung.

Aufgabe 3: Eine Aufgabe zum Handlungsaspekt Argumentieren und Begründen

Ron stimmt im Interview dieser Argumentation direkt zu. Adam erklärt:

Adam: „Wie gesagt: Hier oben hätte ich auch ausgeklammert. Aber ob man das jetzt einfach weglassen kann und nur die $x$-Werte betrachten kann, kann ich nicht sagen. Ich beschäftige ich mich auch nicht jeden Tag damit. Das ist schon ein bisschen her."

Adam zeigt hier allgemein Schwierigkeiten, sich mathematisch auszudrücken. Anstatt vom „Klammern auflösen“ zu sprechen spricht er vom „Ausklammern“. Auch bezeichnet er die Koeffizienten vor dem $x$ als „, $x$-Werte“. Insgesamt ist er sich hinsichtlich der geführten Argumentationskette unsicher und kann in Bezug auf deren Wahrheitsgehalt keine Entscheidung treffen.

Bei der Alternativfrage zum Argumentieren haben sowohl Ron als auch Adam ähnliche Schwierigkeiten gezeigt, trotzdem sie von unserem Kompetenzmodell stark unterschiedlich hinsichtlich der Argumentationskompetenz eingestuft wurden (eine Erklärung könnte wieder bei der geringen Anzahl der gelösten Aufgaben liegen). Ebenso konnte eine Analyse der Erklärungen der falsch gelösten Aufgaben nicht aufdecken, ob es sich tatsächlich um Mängel beim Argumentieren handelt oder ob andere Probleme für das falsche Lösen der Aufgabe ursächlich waren. Im Speziellen das Standardverfahren zum Lösen linearer Gleichungen (was unter den Aspekt des Operierens fällt) müsste im Kontext der Alternativfrage nochmals wiederholt werden.

Insbesondere um hierfür genauere Informationen zu bekommen, sollte dieser Aspekt weitergehend überprüft werden. 
Wiedergeben, Erkennen, Beschreiben

Eine der Aufgaben zum Handlungsaspekt Wiedergeben, Erkennen, Beschreiben, welche die Studierenden im Vorfeld der Untersuchung bearbeitet hatten, ist die folgende Aufgabe 4.

Obwohl vor dem Interview falsch bearbeitet, löst Malin diese Aufgabe im Gespräch richtig. Es könnte sich folglich zuvor um einen Leichtsinnsfehler gehandelt haben.

Ordnen Sie folgende Zahlen nach der Größe:

$A: \frac{6}{100} \quad B: \frac{2}{30} \quad C: \frac{4}{50}$

○ $A<B<C$

○ $B<C<A$

○ $C<A<B$

○ $B<A<C$

(๑) $A<C<B$

Aufgabe 4: Eine erste Aufgabe zum Handlungsaspekt Wiedergeben, Erkennen, Beschreiben

Ella hingegen, die die Aufgabe auch zunächst falsch bearbeitet hat, arbeitet während des Interviews folgendermaßen:

$$
\begin{aligned}
& A: \frac{6}{100}=0,006 \\
& B: \frac{2}{30}=0,075
\end{aligned} \mid C: \frac{4}{50}=0,08
$$

Abbildung 3: Ellas Antwort zum Teil 1 der Alternativfrage Wiedergeben, Erkennen, Beschreiben

Sie beschreibt:

Ella: „Ja. (...) Also da hätte ich das jetzt schriftlich einzeln ausgerechnet, die einzelnen Punkte."

I: „Was heißt ausgerechnet?“

Ella: „Also 6 geteilt durch 100, 2 geteilt durch 30 und 4 geteilt durch 50. Dann hätte ich normale Dezimalzahlen und hätte das dann dementsprechend rausgesucht.“

I: „Ah ja. Zum Beispiel?“

Ella: „Die A sind $\frac{6}{100}$, also (schreibt) sechs Hundertstel sind dasselbe wie 0,006 [I: „Mhm und bei der B?"] und bei der B habe ich zwei Dreißigstel. Zwei geteilt durch Drei sind 0,075 und bei der C: 50 , sind dann (...) 0,8 und dann hätte ich 0,08 . So hätte ich das." 
Ella zeigt hier Schwierigkeiten bei der Verwendung verschiedener Darstellungsweisen von Bruchzahlen. Sie arbeitet nicht mit der gegebenen Darstellung und macht Fehler bei der Umwandlung in Dezimalbruchdarstellung. Ihre Schwierigkeiten werden von unserem Kompetenzsystem daher richtig eingestuft.

Die folgende Alternativfrage bearbeitet sie allerdings wieder korrekt.
$\frac{1}{5}$ entspricht...
○ $\frac{1}{5} \%$
O $50 \%$
$\odot 20 \%$
O $15 \%$
O $95 \%$

Aufgabe 5: Eine zweite Aufgabe zum Handlungsaspekt Wiedergeben, Erkennen, Beschreiben

Diese Frage konnte von allen Teilnehmer*innen, die diese vorgelegt bekamen (Ella, Malin, Miro, Adam), richtig beantwortet werden. Es blieb damit weiterhin unklar, warum Malin schlechter als die anderen abgeschlossen hatte. Dazu müsste auch dieser Aspekt noch weitergehend untersucht werden.

Eine weitere Alternativfrage wurde obig genannten vier Teilnehmer*innen vorgelegt:

Alternativfrage: $\quad \frac{1}{5} \mathrm{~km}=\cdots \mathrm{mm}$

Aufgabe 6: Eine dritte Aufgabe zum Handlungsaspekt Wiedergeben, Erkennen, Beschreiben

Nur Ella zeigte hierbei Probleme, Malin, Miro und Adam beantworteten diese richtig.

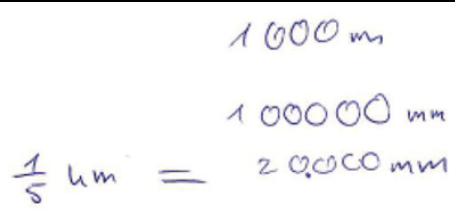

Abbildung 4: Ellas Antwort zum Teil 2 der Alternativfrage Wiedergeben, Erkennen, Beschreiben 
Obwohl also Malin im Vorfeld des Interviews die schlechteste Rückmeldung zum Handlungsaspekt „Wiedergeben, Erkennen, Beschreiben“ bekommen hatte, war es Ella, die im Interview am meisten Schwierigkeiten zeigte. Zwar lassen sich ihre Schwierigkeiten in den Bereich „Wiedergeben, Erkennen, Beschreiben“ einordnen, allerdings bleibt zu klären, warum Malin zunächst Probleme mit den Aufgaben hatte, im Interview dann aber nicht mehr. Insgesamt zeigen vor allem Ella (gutes Abschneiden vor dem Interview, schlechtes Abschneiden während des Interviews) und Malin (eher mäßiges Abschneiden vor dem Interview, gutes Abschneiden während des Interviews) starke Abweichungen bezüglich dieses Handlungsaspekts im Vergleich der Vorarbeit und der Tätigkeit während des Interviews. Die Ursachen sollten an dieser Stelle noch weiter erforscht werden.

\section{Operieren und Berechnen}

Malin hat folgende Frage zum Operieren und Berechnen zunächst falsch bearbeitet:

Vereinfachen Sie soweit wie möglich:

$$
\frac{\frac{a}{a+1}-\frac{b}{b+1}}{\frac{a-b}{a+b}}
$$

Gehen Sie davon aus, dass alle Nenner ungleich 0 sind.

$$
\begin{aligned}
& \odot \frac{a+b}{(a+1)(b+1)} \\
& \odot \frac{2 a+b}{(a+1)(b+1)} \\
& \odot \frac{a+b}{(a+1)(2 b+1)} \\
& \circ \frac{a+b}{(a-1)(b+1)} \\
& \frac{1}{a+b}
\end{aligned}
$$

Aufgabe 7: Eine erste Aufgabe zum Handlungsaspekt Operieren und Berechnen

Im Interview versucht er sie erneut zu lösen:

$$
\begin{aligned}
& \left(2 \frac{a}{a+1}-\frac{b}{b+1} \cdot\left(\frac{a-b}{a+b}\right)\right. \\
& \frac{a^{2}-a b}{(a+1)(a+b)}-\left(-\frac{b^{2}+a b}{(b+1) \cdot(a+b)}\right)=\frac{a^{2}+b^{2}+2 a b}{(a+1) \cdot(a+b)}=\frac{(a+b)^{2}}{a^{2}}
\end{aligned}
$$

Abbildung 5: Lösungsversuch zur ersten Aufgabe von Malin 
Schon die erste Zeile des Lösungswegs von Malin zeigt Mängel: Zum einen fehlen die Klammern um die Differenz der ersten beiden Brüche. Zum anderen wird fälschlicherweise mit dem Nenner des Doppelbruchs multipliziert anstatt mit dem Kehrbruch des Nenners. In der zweiten Zeile werden die unterschiedlichen Nenner nicht beachtet und auch das Minus vor dem zweiten Bruch nicht richtig umgesetzt.

Malin zeigt hier offensichtliche Probleme beim Rechnen mit Brüchen. Auch Basil wird zur Bearbeitung dieser Aufgabe im Interview befragt:

Basil: „Habe ich die bearbeitet, ist das so? Okay. (...) Das Lustige ist - bei diesen Aufgaben - die Herangehensweise. Ich habe darauf, glaub ich, erstmal gegoogelt. Und zwar habe ich mir das auf zwei Brüche aufgeteilt gehabt. Ich habe erst mal nach diesem Format geschaut. Wenn ich das jetzt einfach als a, b, c, d betiteln würde, unter dem Bruchstrich von oben nach unten gehend, wie ich das multiplizieren würde. Was oben (...) im Zähler stehen würde und unten im Nenner (...) und ich weiß, also ich kann mich schon erinnern, dass ich die gemacht habe. Das Problem ist, ich weiß zu hundert Prozent, dass ich die auch nicht richtig gemacht habe und wenn ich jetzt halt (abgebrochen).“

I: „Warum weißt du das zu hundert Prozent, dass du das nicht richtig gemacht hast?“

Basil: „Weil das was mit Variablen zu tun hat. Da bin ich, also ich lerne nach dem Prinzip, dass ich mich an Übungsaufgaben teilweise entlanghangele, also so als Leitfaden. Schaue, ,Wie wurde die gelöst?' und gehe dann da entlang und danach ähnliche Aufgaben löse. Also erstmal ist es eine Ewigkeit her und zweitens, wenn ich eine einfache Aufgabe, also nur auf verschiedene Themenbereiche aus verschiedenen Aufgabenpools, wenn ich die dann einzeln lösen muss und teilweise dann größere Pausen dazwischen sind, geschweige denn keinen Lösungsansatz habe, dann hab ich nicht diesen Leitfaden, damit ich mich entlanghangeln kann und wenn die Aufgabe mir aber so vorkommt, dann ist halt meine Herangehensweise: Ich schaue mir jetzt die Lösung an und schaue, was für mich da am meisten Sinn machen würde, bzw. experimentiere ich ein bisschen, wie ich rechnen könnte. (liest) Das ist nämlich das Problem. Ich habe mir hier genau die Rechenweise nicht wirklich angeschaut, in letzter Zeit. Das ist jetzt ein bisschen peinlich. (...) Geschweige denn Mathe. (...) Also wenn ich mich richtig erinnere, ist das $\frac{a \cdot d}{b \cdot c}$. Ich weiß nicht mehr, ob das schon stimmt. (...), also nach dem Prinzip. Dementsprechend müsste ich den (...) Nenner und das unter dem Bruchstrich stehende im Bruch mal den (...) es macht halt keinen Sinn, weil da ein Minus dazwischen ist (...) Das würde jetzt zu viel Zeit brauchen, um das zu machen und in einer halben Stunde (...) Erst musste ich gucken, was für mich am sinnvollsten war und ich hätte hier jetzt nicht wirklich den Lösungsweg gehabt. Ich würde es jetzt vielleicht probieren. (...) Wie geht das? (...) Also ich würde jetzt verschiedene Möglichkeiten probieren. Aber da weiß ich auch nicht. Ich bin so eine Person, ich kann zeichnen, aber ich missachte teilweise die Regeln, deswegen habe ich da auch vieles falsch gehabt. Und zwar: Wenn ich das jetzt in diesem Bruch erstmal alles einzeln betrachten würde und dann die Nenner hochmultipliziere, sprich: $(a \cdot(b+1))-(b \cdot(a+1))$, dann hätte ich oben erstmal verhindert, dass da (...) Nein, aber wenn ich mir die Lösung anschauen, dann ist das entweder weggekürzt oder es geht nicht. (...) Ja. Also ehrlich gesagt: Sie sollten jetzt hier nicht so viel erwarten. (lacht)“" 
Basil macht hier deutlich, dass er Schwierigkeiten hat, sich Rechenverfahren zu merken und folglich diese richtig anzuwenden. Ihm werden durch das falsche Lösen der Aufgabe somit richtigerweise Mängel beim Operieren und Berechnen bescheinigt.

Obwohl Malin und Basil aber in obiger Aufgabe Probleme beim Operieren gezeigt hatten, haben sie die folgende ihnen vorgelegte Alternativaufgabe richtig beantwortet.

Lösen Sie die Gleichung für $x \in \mathbb{R}: \frac{x}{5} \cdot(-10 x+5)+7=\frac{7}{2} x$.

Aufgabe 8: Eine zweite Aufgabe zum Handlungsaspekt Operieren und Berechnen

Dies passt zu ihrem mittelmäßigen Abschneiden hinsichtlich des Handlungsaspekts Operieren in unserem Kompetenzmodell.

\subsection{Diskussion der Ergebnisse und Schlussfolgerungen}

Zusammenfassend ergab die Auswertung der Interviews folgende Ergebnisse hinsichtlich unserer Forschungsfragen:

- Unser Kompetenzmodell scheint für „Mathematisieren“ und „Darstellungen verwenden" sinnvolle Rückmeldungen zu liefern. Schwierigkeiten zeigten sich bei beiden Handlungsaspekten, insbesondere beim Übersetzen einer realen Situation in die Sprache der Mathematik sowie bei der Darstellung von Bruchzahlen auf der Zahlengerade und in Kreisdiagrammen.

- Hinsichtlich „Argumentieren und Begründen“ und „Wiedergeben, Erkennen, Beschreiben" sind die Ergebnisse zu uneinheitlich, hier müsste eine weitere detailliertere Untersuchung stattfinden. Beim „Argumentieren und Begründen“ wurde durch unsere explorative Studie noch nicht sichtbar, ob sich die gefundenen Probleme der Lernenden nicht auch auf Mängel mit dem Handlungsaspekt „Operieren und Berechnen“ zurückführen lassen, wenn etwa die Umrechnung verschiedener Darstellungsweisen von Bruchzahlen sowie die Umwandlung von Größen nicht beherrscht wird. Die gleichen Schwierigkeiten bei der Bewertung einer vorgegebenen mathematischen Argumentation traten auch bei Studierenden auf, die durch unser Modell im Vorfeld stärkere Ausprägungen dieses Handlungsaspekts zugewiesen bekamen. Dieses ambivalente Bild zeigt sich auch bei „Wiedergeben, Erkennen, Beschreiben“. Auch hier konnten die im Vorfeld zurückgemeldeten Ausprägungen in den Interviews nicht bestätigt werden. 
- Für „Operieren und Berechnen“ gab unser Kompetenzmodell hilfreiche Einschätzungen an, denn die von den Teilnehmer*innen gezeigten Probleme lagen tatsächlich im Bereich des mathematischen Operierens. Das Arbeiten mit Doppelbrüchen und die korrekte Verwendung der Vorzeichenregeln stellten sich hierbei als Schwierigkeiten heraus.

- Darüber hinaus zeigten sich die beiden Handlungsaspekte „Wiedergeben, Erkennen, Beschreiben“ und „Operieren und Berechnen“ auch bei den anderen Handlungsaspekten jeweils als wesentliche Grundfähigkeiten, und es sollte in Betracht gezogen werden, diesen eine gesonderte Rolle zukommen zu lassen. Ein Vorschlag wäre daher, bei den Handlungsaspekten „Darstellungen verwenden“, „Argumentieren und Begründen“ sowie „Mathematisieren“ die anderen beiden Aspekte generell vorauszusetzen. ${ }^{2}$ Ein passendes Modell könnte dann folgendermaßen aussehen:

Tabelle 6: Vorschlag für eine Weiterentwicklung des Kompetenzmodells im optes-Kurs

\begin{tabular}{|c|c|c|c|c|}
\hline \multirow{10}{*}{ 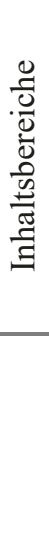 } & LoK 1: Arithmetik & & & \\
\hline & $\begin{array}{l}\text { LoK 2: Gleichungen/ } \\
\text { Ungleichungen }\end{array}$ & & & \\
\hline & $\begin{array}{l}\text { LoK 3: Potenzen, } \\
\text { Wurzeln, Logarith- } \\
\text { men }\end{array}$ & & & \\
\hline & LoK 4: Funktionen & & & \\
\hline & LoK 5: Geometrie & & & \\
\hline & LoK 6: Trigonometrie & & & \\
\hline & & $\begin{array}{c}\text { Darstellungen } \\
\text { verwenden }\end{array}$ & Mathematisieren & $\begin{array}{l}\text { Argumentieren } \\
\text { und Begründen }\end{array}$ \\
\hline & & \multicolumn{3}{|c|}{ Operieren und berechnen } \\
\hline & & \multicolumn{3}{|c|}{ Wiedergeben, erkennen, beschreiben } \\
\hline & & \multicolumn{3}{|c|}{ Handlungsaspekte } \\
\hline
\end{tabular}

2 Im HarmoS-Modell wird der Handlungsaspekt Wissen, Erkennen und Beschreiben als „mathematisches Grundwissen“ charakterisiert, was bereits seine grundlegende Bedeutung für die anderen Handlungsaspekte unterstreicht (Linneweber-Lammerskitten, Wälti und Moser Opitz 2009, S. 15). 
Diese vorgeschlagene Änderung des Kompetenzmodells würde allerdings in der praktischen Ausführung keine Konsequenzen mit sich bringen. Lediglich soll den Lernenden damit deutlich gemacht werden, dass ohne eine ausreichende Ausprägung der Kompetenzen „Operieren und Berechnen“ sowie „Wiedergeben, Erkennen, Beschreiben“ Aufgaben zu „Darstellungen verwenden“, „Mathematisieren“ und „Argumentieren und Begründen“ nicht gelöst werden können.

\section{Literatur}

Blömeke, S. (2016). Der Übergang von der Schule in die Hochschule: Empirische Erkenntnisse zu mathematikbezogenen Studiengängen. In A. Hoppenbrock, R. Biehler, R. Hochmuth \& H.-G. Rück (Hrsg.), Lehren und Lernen von Mathematik in der Studieneingangsphase. Herausforderungen und Lösungsansätze (S. 3-14). Wiesbaden: Springer.

Bortz, J. \& Döring, N. (2006). Forschungsmethoden und Evaluation (4. Auflage). Heidelberg: Springer.

cosh-Katalog (2014). Mindestanforderungskatalog Mathematik (Version 2.0) der Hochschulen Baden-Württembergs für ein Studium von WiMINT-Fächern. Verfügbar unter https://lehrerfortbildung-bw.de/u_matnatech/mathematik/bs/bk/cosh/katalog/mak v20b ohne leerseiten.pdf [10.06.2020].

De Guzman, M., Hodgson, B. R., Robert, A. \& Villani, V. (1998). Difficulties in the passage from secondary to tertiary education, Proceedings of the International Congress of Mathematicians, Berlin. Documenta mathematica, extra volume ICM 111, 747-762.

Gueudet, G. (2008). Investigating the secondary-tertiary transition. Educational studies in mathematics, 67(3), 237-254.

Hoppenbrock, A., Biehler, R., Hochmuth, R. \& Rück, H.-G. (Hrsg.) (2016). Lehren und Lernen von Mathematik in der Studieneingangsphase. Herausforderungen und Lösungsansätze. Wiesbaden: Springer.

Kortemeyer, J. (2018). Mathematische Kompetenzen in ingenieurwissenschaftlichen Grundlagenveranstaltungen: normative und empirische Analysen zu exemplarischen Klausuraufgaben aus dem ersten Studienjahr in der Elektrotechnik (Dissertation). Verfügbar unter http://digital.ub.uni-paderborn.de/hs/content/titleinfo/2855023 [10.06. 2020].

Leuders, T. (2014). Modellierungen mathematischer Kompetenzen - Kriterien für eine Validitätsprüfung aus fachdidaktischer Sicht. Journal für Mathematik-Didaktik, 35, 7-48.

Linneweber-Lammerskitten, H., Wälti, B. \& Moser Opitz, E. (2009). HarmoS Mathematik. Wissenschaftlicher Kurzbericht und Kompetenzmodell. Verfügbar unter https://ink. springer.com/article/10.1007/s13138-013-0060-3 [10.06.2020].

Linneweber-Lammerskitten, H. (Hrsg.) (2014). Fachdidaktik Mathematik-Mathematikdidaktik - Grundbindung und Kompetenzaufbau im Unterricht der Sek. I und II. Seelze: Klett u. Kallmeyer.

Roth, J., Bauer, T., Koch, H. \& Prediger, S. (Hrsg.) (2015). Übergänge konstruktiv gestalten: Ansätze für eine zielgruppenspezifische Hochschuldidaktik Mathematik. Wiesbaden: Springer.

Schweizerische Konferenz der kantonalen Erziehungsdirektoren (2011). Grundkompetenzen für die Mathematik - Nationale Bildungsstandards. Verfügbar unter https://edudoc. $\mathrm{ch} / \mathrm{record} / 96784 ? \ln =\mathrm{de}[10.06 .2020]$. 
Weinert, F. (2001). Vergleichende Leistungsmessung in Schulen - eine umstrittene Selbstverständlichkeit. In F. Weinert (Hrsg.), Leistungsmessungen in Schulen (S. 17-32). Weinheim: Beltz.

Open Access Dieses Kapitel wird unter der Creative Commons Namensnennung 4.0 International Lizenz (http://creativecommons.org/licenses/by/4.0/deed. de) veröffentlicht, welche die Nutzung, Vervielfältigung, Bearbeitung, Verbreitung und Wiedergabe in jeglichem Medium und Format erlaubt, sofern Sie den/ die ursprünglichen Autor(en) und die Quelle ordnungsgemäß nennen, einen Link zur Creative Commons Lizenz beifügen und angeben, ob Änderungen vorgenommen wurden.

Die in diesem Kapitel enthaltenen Bilder und sonstiges Drittmaterial unterliegen ebenfalls der genannten Creative Commons Lizenz, sofern sich aus der Abbildungslegende nichts anderes ergibt. Sofern das betreffende Material nicht unter der genannten Creative Commons Lizenz steht und die betreffende Handlung nicht nach gesetzlichen Vorschriften erlaubt ist, ist für die oben aufgeführten Weiterverwendungen des Materials die Einwilligung des jeweiligen Rechteinhabers einzuholen.

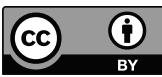

